\title{
Biometric Personal Identification based on Iris Patterns
}

\author{
Yogesh Pandit, Chandan Singh D Rawat \\ V.E.S. Institute of Technology, Department of Electronics and Telecommunication Engineering \\ Chembur, Mumbai 400076 \\ e-mail: yogesh_spandit@yahoo.co.in
}

\begin{abstract}
This paper discusses an analysis of human iris patterns for recognition of biometric system which consists of a segmentation system that is based on the Hough transform, and is able to localize the circular iris and pupil region, occluding eyelids and eyelashes, and reflections. The extracted iris region is then normalized into a rectangular block with constant dimensions to account for imaging inconsistencies. To encode the unique pattern of the iris into a bit-wise biometric template, 1D Log-Gabor filter is used.Finally to match two iris templates hamming distance is used as matching metric. The system performance is analyzed on 312 iris images taken from standard CASIA Iris Interval database version 4. To establish the verification accuracy of iris representation and matching approach, each iris image in the database is matched with all the other iris images in the database and genuine and imposter distribution is found. The performance of the system is implemented by evaluating the Decidability Index (DI), False match rate (FMR), False Non-match rate (FNMR), Genuine Accept Rate (GAR) and Equal error rate (EER).
\end{abstract}

Keywords: Hough Transform, Log-Gabor filter, Hamming distance, Genuine Accept Rate

\section{Introduction}

A biometric system provides automatic recognition of an individual based on some sort of unique feature or characteristic possessed by the individual. Biometric systems have been developed based on fingerprints, facial features, voice, hand geometry, handwriting, the retina, Iris etc.Using a biometric system for person identification (or verification) has been an attractive proposition for two reasons.

i. There is no need to remember a password,

ii. The biological features used are always available with the individual (no need to carry an identification card). Biometric systems work by first capturing a sample of the biological feature (e.g. an eye image). Usually the digitized sample contains a lot of redundancy and need to be represented in a compact form using some kind of mathematical function. The transformed sample is termed as a biometric template. The template can be objectively compared with other templates in the database in order to verify / determine a person's identity.

Iris is a thin circular diaphragm lying between the cornea and the lens of the human eye. A circular aperture known as the pupil is present near the center of iris. Iris controls the amount of light entering through the pupil by adjusting its size. The average diameter of the iris is $12 \mathrm{~mm}$ while the pupil size varies from $10 \%$ to $80 \%$ of the iris diameter [1]. Eye anatomy is shown in Figure 1.1 Formation of the iris begins during the third month of embryonic life [2]. During the first year, the unique pattern on the iris surface is formed. Formation of the unique patterns of the iris is random and not related to any genetic factors . Iris color, though, is dependent on genetics. Iris patterns in our both eyes are independent of each other. Even the identical twins' iris patterns are not correlated. 


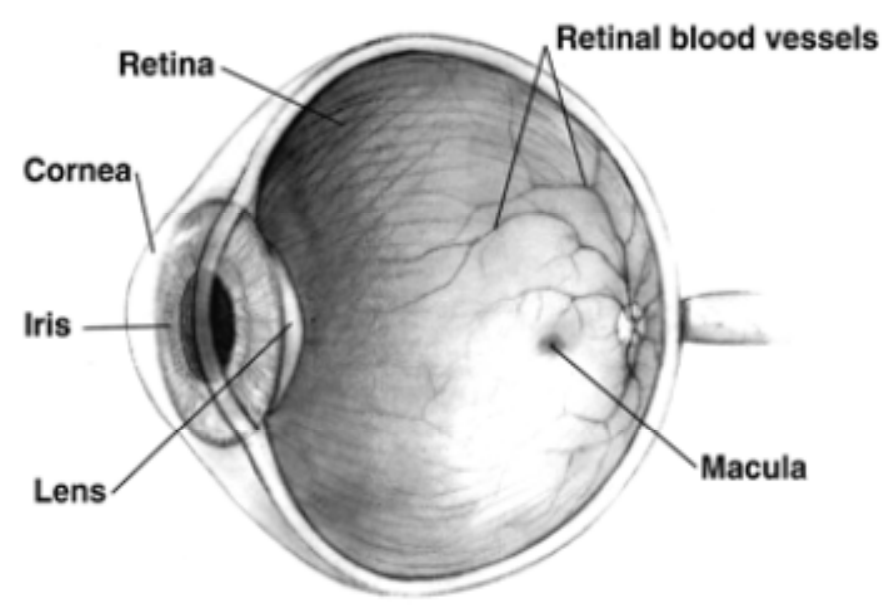

Figure 1.1. Eye anatomy [3]

\section{IRIS Recognition Method}

In 1994, John Daugman obtained the patent for "Biometric personal identification system based on iris analysis". Some other iris recognition systems were also developed by other people, such as Wildes et al. [4], Lim et al. [5] and Boles and Boashash [6]. Iris feature is convenience for a person to prove his/her identity based on him/her biometrics at any place and at any time.The iris pattern remains stable throughout adult life. Iris recognition system includes iris capturing, image pre-processing, iris region localization, iris region normalization, iris feature extraction and pattern matching. Every part is very important for correct recognition person identity. [7]

Block diagram for iris recognition is shown in Figure 2.1

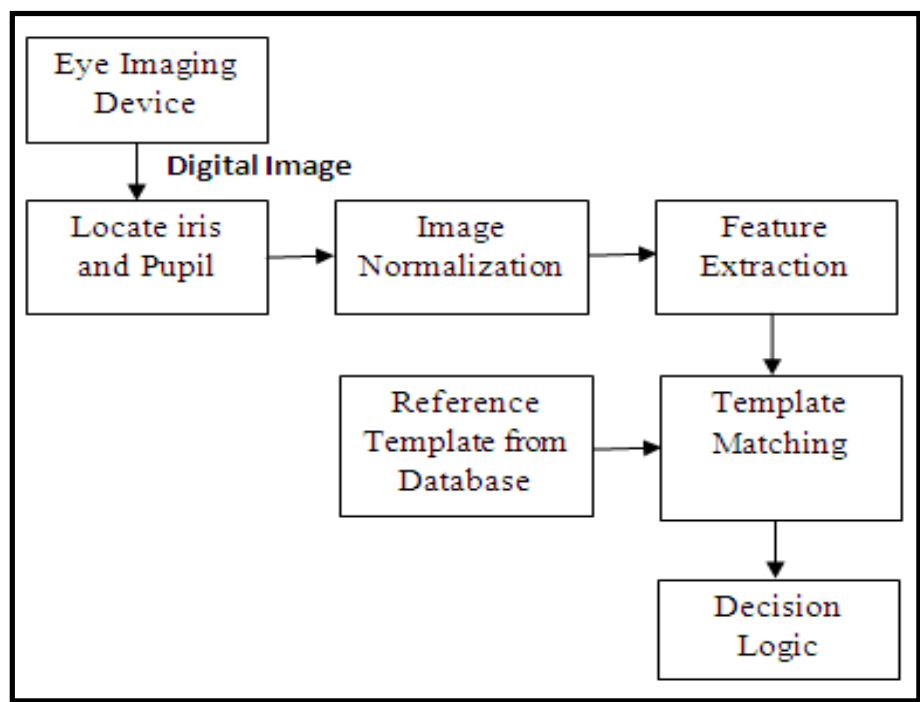

Figure 2.1. Block diagram for Iris recognition

\subsection{Iris localization}

The first step in iris recognition is to locate iris and pupil in an image of eye. In general, the pupil and the iris are not concentric; also pupil radius is not in a fixed ratio to the iris radius [2]. Hence, parameters such as $x-y$ co-ordinates and radius corresponding to both the iris and the pupil are need to know. This stage is termed as "Iris segmentation / iris localization". The 
segmentation stage is critical to the success of an iris recognition system. Iris pattern corrupted due to erroneous segmentation results in poor recognition rates.

The implemented system uses a segmentation algorithm based on the circular Hough transform for detecting the iris and pupil boundaries [8].This involves first employing Canny edge detection to generate an edge map. From the edge map, votes are cast in Hough space for the parameters of circles passing through each edge point. These parameters are the centre coordinates $\mathrm{x}_{\mathrm{c}}$ and $\mathrm{y}_{\mathrm{c}}$ and the radius $\mathrm{r}$, which are able to define any circle according to the equation,

$$
\mathrm{x}_{\mathrm{c}}^{2}+\mathrm{y}_{\mathrm{c}}^{2}-\mathrm{r}^{2}=0
$$

A maximum point in the Hough space will correspond to the radius and centre coordinates of the circle best defined by the edge points.

Figure 2.2 shows results after implementing circular Hough transform.

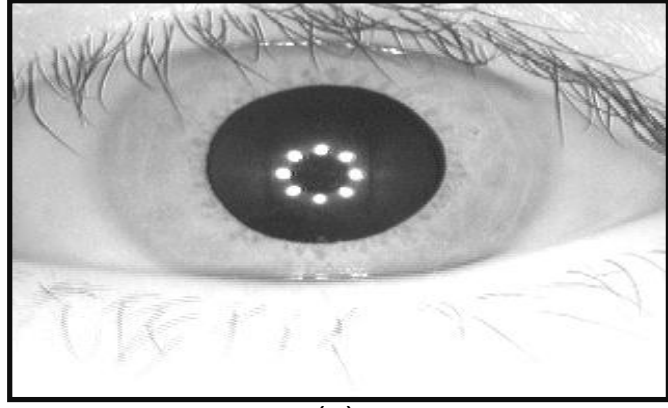

(a)

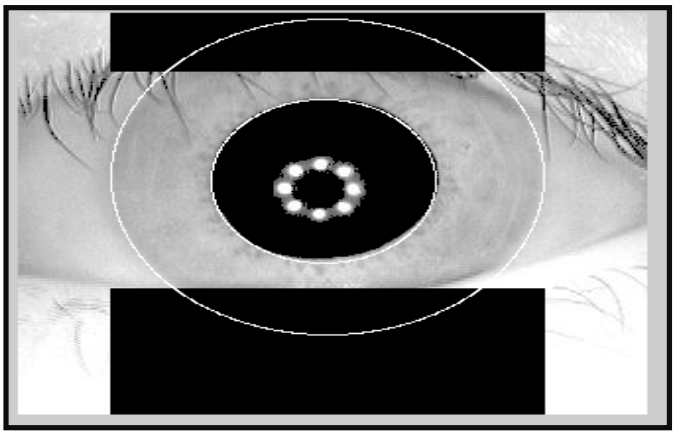

(c)

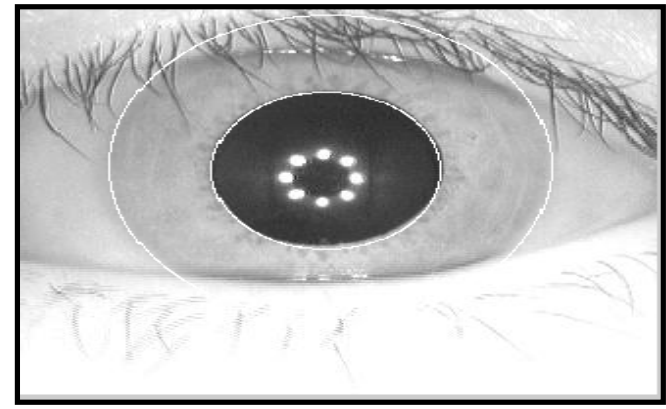

(b)

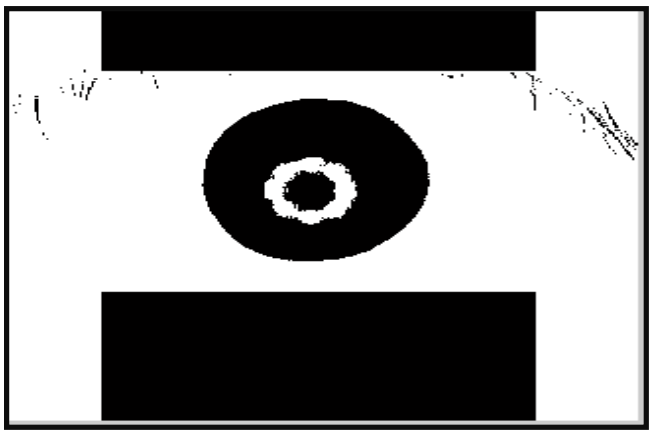

(d)

Figure 2.2. (a) Original eye image from the CASIA database, (b) Two circles overlayed for iris and pupil boundaries, (c) Probable eyelid and eyelash areas isolated (black areas) and (d) Automatic segmentation of the image. Black regions denote detected eyelid and eyelash regions.

\subsection{Iris Normalization}

Once the iris region is successfully segmented from an eye image, the next stage is to transform the iris region so that it has fixed dimensions in order to allow comparisons. The dimensional inconsistencies between eye images are mainly due to the stretching of the iris caused by pupil dilation from varying levels of illumination. Other sources of inconsistency include, varying imaging distance, rotation of the camera, head tilt, and rotation of the eye within the eye socket [1]. The normalization process will produce iris regions, which have the same constant dimensions, so that two images of the same iris under different conditions will have characteristic features at the same spatial location. For this purpose Daugman's Rubber Sheet model is used [9]. The homogenous rubber sheet model devised by Daugman remaps each 
point within the iris region to a pair of polar coordinates $(r, \theta)$ where $r$ is on the interval $[0,1]$ and $\theta$ is angle $[0,2 \pi]$.

The centre of the pupil was considered as the reference point, and radial vectors pass through the iris region.A number of data points are selected along each radial line and this is defined as the radial resolution. The number of radial lines going around the iris region is defined as the angular resolution. Since the pupil can be non-concentric to the iris, a remapping formula is needed to rescale points depending on the angle around the circle.

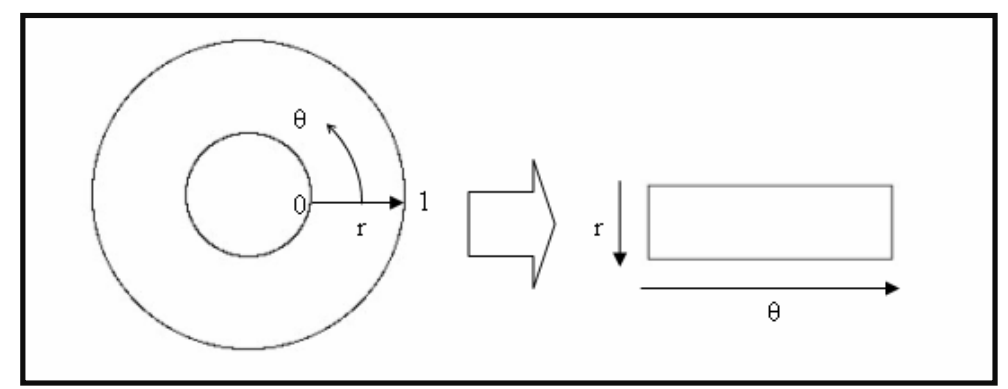

Figure 2.3. Visual representation of Daugman's rubber sheet model. [10]

$$
\begin{aligned}
& I(x(r, \theta), y(r, \theta) \rightarrow I(r, \theta)) \\
& x(r, \theta)=(1-r) x_{p}(\theta)+r x_{1}(\theta) \\
& y(r, \theta)=(1-r) y_{p}(\theta)+r y_{1}(\theta)
\end{aligned}
$$

Where $I(x, y)$ is the iris region image, $(x, y)$ are the original Cartesian coordinates, $(r, \theta)$ are the corresponding normalised polar coordinates, $\left(x_{p}, y_{p}\right)$ and $\left(x_{I}, y_{I}\right)$ and are the coordinates of the pupil and iris boundaries along the $\theta$ direction. The rubber sheet model takes into account pupil dilation and size inconsistencies in order to produce a normalised representation with constant dimensions. Figure 2.4 shows normalized iris image.

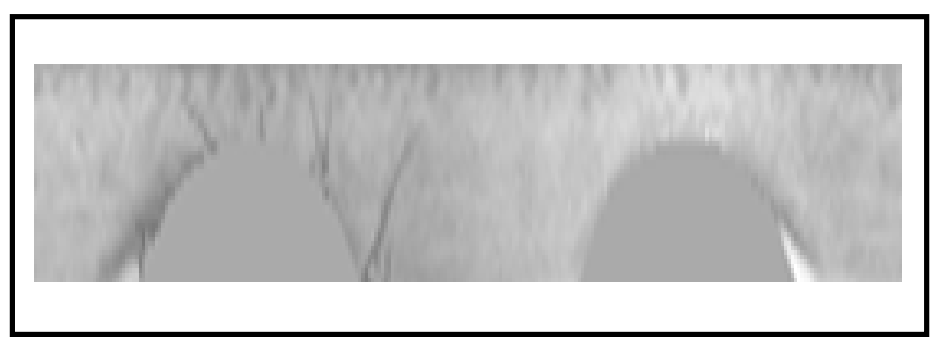

Figure 2.4. The normalized iris image

\subsection{Feature Encoding}

In order to provide accurate recognition of individuals, the most discriminating information present in an iris pattern must be extracted. Only the significant features of the iris must be encoded so that comparisons between templates can be made. For this purpose Gabor filters are used to provide optimum conjoint representation of a signal in space and spatial frequency. But a disadvantage of the Gabor filter is that the even symmetric filter will have a DC component whenever the bandwidth is larger than one octave. However, zero DC components can be obtained for any bandwidth by using a Gabor filter which is Gaussian on a logarithmic scale, this is known as the Log-Gabor filter. The frequency response of a Log-Gabor filter is given as, [8] 
$G(f)=\exp \left(\frac{-\left(\log \left(\frac{f}{f f}\right)\right)^{2}}{2\left(\log \left(\frac{\sigma}{f o}\right)\right)^{2}}\right)$

where fo represents the centre frequency, and $\sigma$ gives the bandwidth of the filter.

\subsection{Matching}

For matching, the Hamming distance is chosen as a metric for recognition, since bitwise comparisons are necessary. In comparing the bit patterns $X$ and $Y$, the Hamming distance, $\mathrm{HD}$, is defined as the sum of disagreeing bits (sum of the exclusive-OR between $X$ and $Y$ ) over $\mathrm{N}$, the total number of bits in the bit pattern. [8]

$$
\mathrm{HD}=\frac{1}{\mathrm{~N}} \sum_{\mathbf{j}=1}^{\mathrm{N}} \mathbf{X}_{\mathbf{j}}(\mathbf{X O R}) \mathbf{Y}_{\mathbf{j}}
$$

The Hamming distance algorithm employed also incorporates noise masking, so that only significant bits are used in calculating the Hamming distance between two iris templates. Now when taking the Hamming distance, only those bits in the iris pattern that corresponds to ' 0 ' bits in noise masks of both iris patterns will be used in the calculation. The Hamming distance will be calculated using only the bits generated from the true iris region, and this modified Hamming distance formula is given as [8],

$$
\text { HD }=\frac{\text { Template } 1 \oplus \text { Template2.Mask1'.Mask2' }}{\text { Template-(Mask1+mask2) }}
$$

\section{Metric Used For Performance Evaluation}

The performance of the implemented system is evaluated on the following biometric metric parameters,

\subsection{Decidability Index}

The key objective of an iris recognition system is to be able to achieve a distinct separation of intra-class and inter-class Hamming distance distributions. A separation Hamming distance value can be chosen which allows a decision to be made when comparing two templates. If the Hamming distance between two templates is less than the separation point, the templates were generated from the same iris and a match is found. Otherwise if the Hamming distance is greater than the separation point the two templates are considered to be generated from different irises.

The distance between the minimum Hamming distance value for inter-class comparisons and maximum Hamming distance value for intra-class comparisons could be used as a metric to measure separation; however, this is not a very accurate measure since outliers will corrupt the value calculated, and the measure is dependent on the number of iris templates compared. A better metric is Decidability index (DI) [2], which takes into account the mean and standard deviation of the intra-class and inter-class distributions.

Decidability index $\mathrm{d}^{\prime}$ is a distance measured in standard deviations and is a function of the magnitude of difference between the mean of the intra-class distribution $\mu_{\mathrm{s}}$ and the mean of the inter-class distribution $\mu_{\mathrm{d}}$, and also the standard deviation of the intra-class and inter-class distributions $\sigma_{\mathrm{s}}^{2}$ and $\sigma_{\mathrm{d}}^{2}$ respectively and is given by, [2]

$$
d^{\prime}=\frac{\left|\mu_{s-} \mu_{d}\right|}{\sqrt{\frac{\left(\sigma_{s}^{2}+\sigma_{d}^{2}\right)}{2}}}
$$

The higher the decidability index, the greater the separation of intra-class and interclass distributions which allows for more accurate recognition.

\subsection{FMR, FNMR, GAR}

The degree of similarity between two iris sets is indicated by a similarity score such as a genuine score and an impostor score. An impostor score that exceeds the threshold $\eta$ results in 
a false match, while a genuine score that falls below the threshold $\eta$ results in a false nonmatch.

The False Match Rate (FMR) of a biometric system can therefore be defined as the fraction of impostor scores exceeding the threshold $\eta$. Similarly, the False Non match Rate (FNMR) of a system may be defined as the fraction of genuine scores falling below the threshold $\eta$ [2]. If $\mathrm{T}^{\mathrm{I}}$ and $\mathrm{T}^{\mathrm{G}}$ are total number of Imposter and Genuine pairs then FMR and FNMR are given by following equations.

$$
\begin{aligned}
& F M R(t)=\frac{1}{T^{I}} \sum_{i=0}^{t} \operatorname{Imposter}(i) \\
& \operatorname{FNMR}(t)=\frac{1}{T^{G}} \sum_{i=t}^{0.5} \operatorname{Genuine}(i)
\end{aligned}
$$

The Genuine Accept Rate (GAR) is the fraction of genuine scores exceeding the threshold $\eta$. Therefore, $\quad G A R=1-F N M R$.

\subsection{Equal Error Rate}

The Equal Error Rate (EER) refers to that point in a Detection Error Tradeoff (DET) curve where False Match Rate (FMR) equals the False Non Match Rate (FNMR), a lower the EER value, better the performance of system. [2]

\section{Simulation Results and Discussion}

The experiment is performed on CASIA-Iris-Interval Version 4 database using MATLAB 7.0.

\subsection{Iris Image Database}

Iris images of CASIA-Iris-Interval [10] were captured using a specially developed close up iris camera having circular NIR LED array, with suitable luminous flux for iris imaging. CASIA-Iris-Interval is well-suited for studying the detailed texture features of iris images. The images were acquired using CASIA close-up iris camera in an indoor environment. The resolution of these images is $320 \times 280$ pixels. In this paper, 312 iris images of 39 individuals with 8 images per person are selected. The sample images of the database are shown in figure 4.1

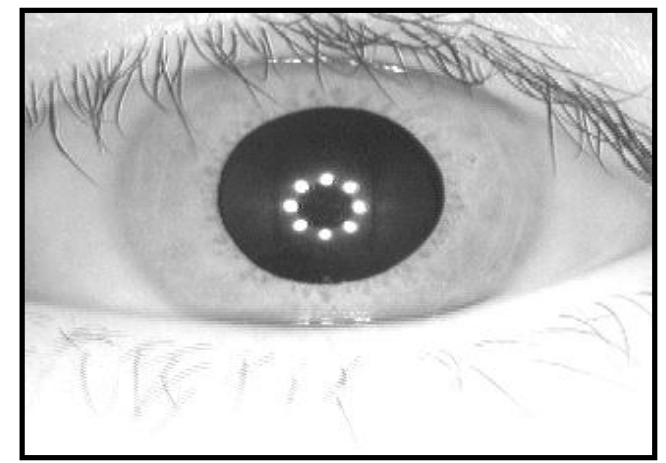

S1001L01.jpg

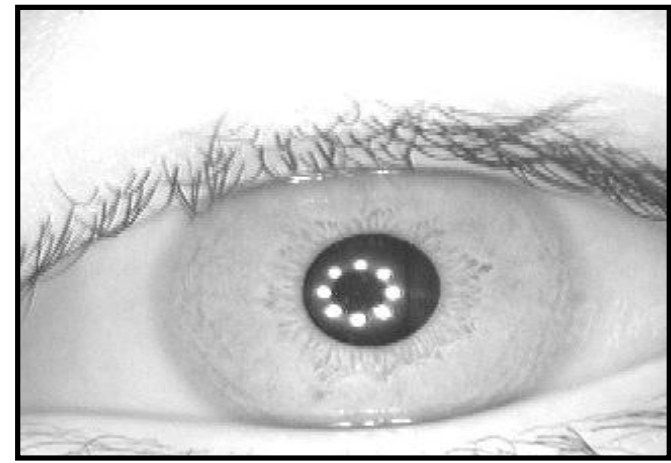

S1002L06.jpg

Figure 4.1. Sample Images of CASIA-IRIS-INTERVAL database [10]

\subsection{Genuine and Imposter Distribution}

The occurrences of genuine and imposter distributions at different value of thresholds are resulted in figure 4.2 (a) which shows that there is less interference between the genuine and imposter which helps to improve the system performance in terms of identification of the 
individual. Figure 4.2 (b) shows the separation of FMR and FNMR at different thresholds. More the separation between two indicates the better identification results.

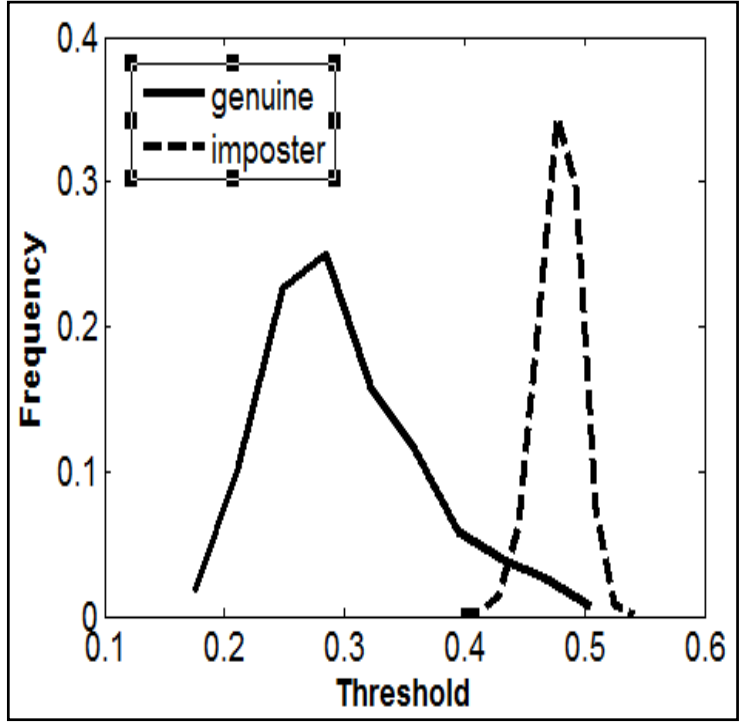

(a)

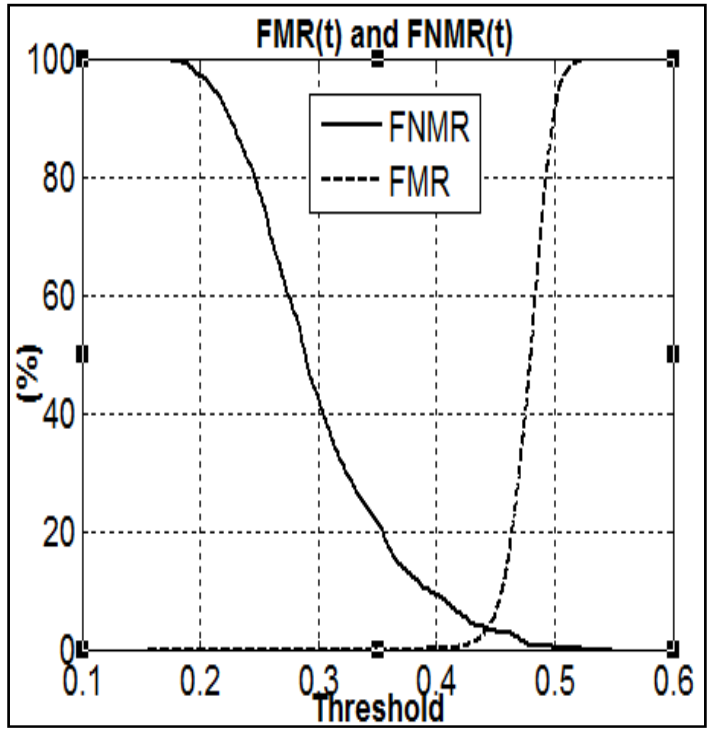

(b)

Figure 4.2. (a) Occurrences of Genuine and imposter Distribution at different thresholds; (b) Occurrences of FMR and FNMR at different thresholds.

\subsection{Receiver Operating Characteristic and Detection Error Tradeoff:}

A Receiver Operating Characteristic (ROC) curve shown in figure 4.3( a) is a plot of Genuine Acceptance Rate against False Match Rate for all possible system operating points and measures the overall performance of the system. In this implemented system the value of Genuine Acceptance rate is $96 \%$ for $0.01 \%$ of FMR value. Another resulted parameter is the EER which is the point in a Detection Error Tradeoff (DET) curve shown in figure 4.3(b) where the value of FMR equals the value of FNMR.

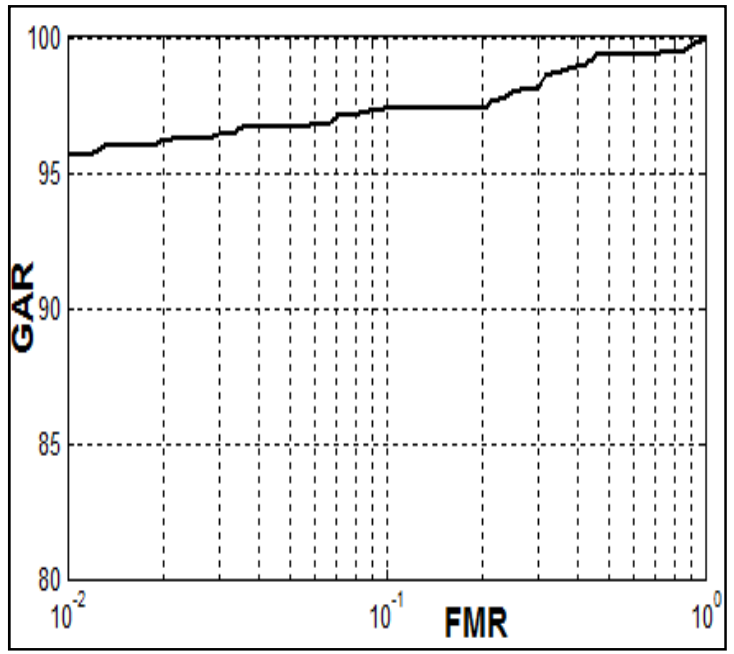

(a)

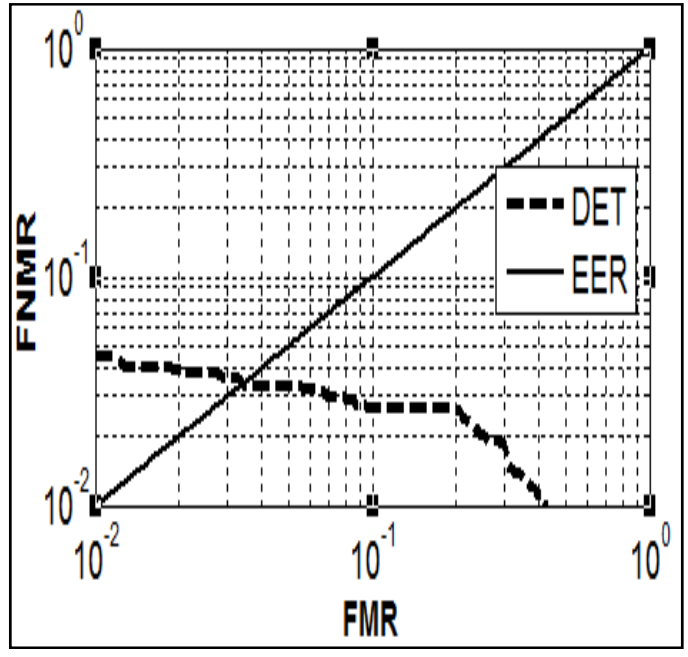

(b) 


\section{Conclusion}

The implemented iris recognition system is tested on CASIA-IRIS-Interval Version4 database of greyscale eye images.The iris from each image is localized by an automatic segmentation algorithm using circular Hough transform. The dimensional inconsistencies between iris regions is eliminated by implementing a Daugman's rubber sheet model.Feature encoding in creation of iris templates is done with 1D Log-Gabor filters.Finally Hamming distance is used as a matching metric. Implemented iris recognition system is able to achieve a distinct separation of genuine and imposter Hamming distance distributions. The performance of implemented system is evaluated by finding out genuine and imposter distribution, Genuine accept rate, Equal error rate and Decidability index.

The implemented system gives 96\% GAR at $0.01 \%$ FMR, 0.0277 EER with 65.021

\section{References}

[1] J Daugman. How iris recognition works. Proceedings of 2002 International Conference on Image Processing, 2002. Vol. 1.

[2] Anil K. Jain (Michigan State University, USA), Patrick Flynn (University of Notre Dame, USA), Arun A. Ross (West Virginia University, USA) "Handbook of Biometrics". 4th, July, 2007.

[3] E Wolff. "Anatomy of the Eye and Orbit". 7th edition. H. K. Lewis \& Co. Itd. 1976.

[4] R Wildes. Iris recognition: an emerging biometric technology. Proceedings of the IEEE. 1997; 85.

[5] S Lim, K Lee, O Byeon and T Kim. "Efficient iris recognition through improvement of feature vector and classifier". ETRI Journal. 2001; 23(2).

[6] W Boles and B Boashash. "A human identification technique using images of the iris and wavelet transform". IEEE Transactions on Signal Processing. 1998; 46(4).

[7] B Boashash. An Efficient Iris Localization Algorithm. 29th National Radio Science Conference, Faculty of Engineering/, April 10 \& 12, 2012, Colorado,

[8] Libor Masek. "Recognition of Human Iris Patterns for Biometric Identification". Bachelor of Engineering Thesis, the, University of Western, Australia 2003

[9] J Daugman. "Biometric personal identification system based on iris analysis". U.S. Patent 5291560 , Mar 1, 1994.

[10] CASIA Iris Image Database version 4, http://biometrics.idealtest.org 